\title{
Physically constrained maximum likelihood mode filtering
}

\author{
Joseph C. Papp, ${ }^{\text {a) }}$ James C. Preisig, and Andrey K. Morozov \\ Woods Hole Oceanographic Institution, MS 44, Woods Hole, Massachusetts 02543
}

(Received 28 September 2009; revised 24 January 2010; accepted 27 January 2010)

\begin{abstract}
Mode filtering is most commonly implemented using the sampled mode shapes or pseudoinverse algorithms. Buck et al. [J. Acoust. Soc. Am. 103, 1813-1824 (1998)] placed these techniques in the context of a broader maximum a posteriori (MAP) framework. However, the MAP algorithm requires that the signal and noise statistics be known a priori. Adaptive array processing algorithms are candidates for improving performance without the need for a priori signal and noise statistics. A variant of the physically constrained, maximum likelihood (PCML) algorithm [A. L. Kraay and A. B. Baggeroer, IEEE Trans. Signal Process. 55, 4048-4063 (2007)] is developed for mode filtering that achieves the same performance as the MAP mode filter yet does not need a priori knowledge of the signal and noise statistics. The central innovation of this adaptive mode filter is that the received signal's sample covariance matrix, as estimated by the algorithm, is constrained to be that which can be physically realized given a modal propagation model and an appropriate noise model. Shallow water simulation results are presented showing the benefit of using the PCML method in adaptive mode filtering. (C) 2010 Acoustical Society of America.
\end{abstract}

[DOI: $10.1121 / 1.3327799$ ]

PACS number(s): 43.60.Mn [EJS]

Pages: 2385-2391

\section{INTRODUCTION}

Buck et al. ${ }^{1}$ presented a unified framework for mode filtering using a model of the underwater environment containing propagating modes plus noise. Nonadaptive linear mode filters such as the sampled mode shapes and pseudoinverse filters were analyzed and the MAP filter was presented to make use of signal and noise statistics and showed a significant performance improvement. However, the statistics are required to be known a priori. This paper proposes an adaptive mode filter based on the minimum power distortionless response (MPDR) beamformer that uses pressure field statistics estimated from the data. However, in a nonstationary environment there are often an insufficient number of snapshots available to accurately estimate the required statistics. Kraay ${ }^{2}$ presented a physically constrained, maximum likelihood (PCML) method for using knowledge of the spatial environment to estimate the statistics using fewer snapshots. This paper adapts the PCML method to the underwater mode estimation problem using adaptive mode filters based on the MPDR and MAP frameworks.

The remaining sections of the introduction introduce the signal and noise model used in this paper and describe several established mode filtering techniques-the sampled mode shapes, pseudoinverse, diagonally weighted pseudoinverse, reduced rank pseudoinverse, and maximum a posteriori (MAP) mode filters. Section II describes the MPDR mode filter and a diagonal weighting technique to address the snapshot deficiency problem. Section III outlines the PCML algorithm developed by Kraay for a spatial beamformer. Section IV describes the adaptation of the PCML algorithm for the mode estimation problem. Lastly, Sec. V presents the

\footnotetext{
a) Author to whom correspondence should be addressed. Electronic mail: jcpapp@mit.edu
}

performance of the various mode filters in a shallow water simulation and Sec. VI summarizes the main conclusions of this paper.

\section{A. Modes as basis functions}

Modes are physically motivated, orthogonal basis functions for the vertical sound field. They are derived from solutions to the wave equation and are dependent on frequency and environmental conditions, such as water depth, temperature, salinity, and bottom properties. ${ }^{3}$ Equation (1) shows the pressure field as a sum of modes, where $\mathbf{p}(z, f)$ is the complex acoustic pressure at frequency $f$ and depth $z, d_{m}$ is the mode coefficient of the $m^{\text {th }}$ mode, and $\boldsymbol{\psi}_{m}(z, f)$ is the $m$ th mode shape as a function of depth and frequency,

$$
p(z, f)=\sum_{m} d_{m}(f) \psi_{m}(z, f)
$$

\section{B. Signal and noise model used}

Equation (2) shows the signal model used to represent the sound pressure field,

$$
\left[\begin{array}{c}
p\left(z_{1}\right) \\
\vdots \\
p\left(z_{N}\right)
\end{array}\right]=\left[\begin{array}{ccc}
\psi_{1}\left(z_{1}\right) & \cdots & \psi_{M}\left(z_{1}\right) \\
\vdots & \ddots & \vdots \\
\psi_{1}\left(z_{N}\right) & \cdots & \psi_{M}\left(z_{N}\right)
\end{array}\right]\left[\begin{array}{c}
d_{1} \\
\vdots \\
d_{M}
\end{array}\right]+\left[\begin{array}{c}
n\left(z_{1}\right) \\
\vdots \\
n\left(z_{N}\right)
\end{array}\right],
$$

where $n(z)$ is the noise received by the sensor at depth $z$. The functional dependence of all quantities on frequency has been dropped for notational convenience. In this paper, the mode shapes, $\psi$, are assumed known a priori and the goal is to estimate the complex mode coefficients $d$ given measurements of the pressure field $\mathbf{p}$. Written in vector notation, the above equation becomes 


$$
\mathbf{p}=\psi \mathbf{d}+\mathbf{n}
$$

where $\boldsymbol{\psi}$ is the matrix of sampled mode shapes at a particular frequency

$$
\boldsymbol{\psi}=\left[\begin{array}{ccc}
\psi_{1}\left(z_{1}\right) & \cdots & \psi_{M}\left(z_{1}\right) \\
\vdots & \ddots & \vdots \\
\psi_{1}\left(z_{N}\right) & \cdots & \psi_{M}\left(z_{N}\right)
\end{array}\right]
$$

and $\mathbf{d}$ is a vector of complex-valued mode amplitudes and is assumed to be zero mean. A spatially white (SW) noise model is used in this paper. The SW noise model assumes the noise is complex valued and zero mean, with the noise at each hydrophone uncorrelated with noise at all of the other hydrophones. Assuming that the noise has save variance at each sensor, the covariance matrix for this type of noise is

$$
\mathbf{R}_{\mathbf{n}}=\sigma^{2} \mathbf{I} \text {. }
$$

Often noise is contained in the propagating modes as well, and this type of noise is described by the Kuperman-Ingenito model. ${ }^{4}$ The goal of mode filtering, however, is to simply estimate the coefficients of the received energy in each mode and not to distinguish between signal and noise contained in the modes. Determining what part of the mode amplitude is from signal and what part is from noise is an application specific problem. Therefore, only spatially white noise will be considered in this paper.

\section{Established mode filtering techniques}

This section outlines several established mode filtering techniques. Mode filters estimate the complex-valued amplitude of a particular mode at a particular frequency given the measured vertical pressure field and knowledge of the mode shapes. Further information about the mode filtering techniques can be found in Ref. 1. Equation (6) expresses the estimated mode amplitudes as a function of the vertical pressure field and a linear mode filter, $\mathbf{H}$.

$$
\hat{\mathbf{d}}=\mathbf{H p} \text {. }
$$

The remaining part of this subsection lists several choices for $\mathbf{H}$ that can be used in estimating the mode amplitudes.

(a) Sampled mode shape (SMS) mode filter.

$$
\mathbf{H}_{\mathrm{SMS}}=\left[\begin{array}{ccc}
\psi_{1}\left(z_{1}\right) & \cdots & \psi_{1}\left(z_{N}\right) \\
\vdots & \ddots & \vdots \\
\psi_{M}\left(z_{1}\right) & \cdots & \psi_{M}\left(z_{N}\right)
\end{array}\right]=\psi^{H} .
$$

(b) Pseudoinverse (PI) mode filter.

$$
\mathbf{H}_{P I}=\left(\boldsymbol{\psi}^{H} \boldsymbol{\psi}\right)^{-1} \boldsymbol{\psi}^{H} .
$$

(c) Diagonal weighting on pseudoinverse mode filter.

$$
\mathbf{H}_{\mathrm{DW}}=\left(\boldsymbol{\psi}^{H} \boldsymbol{\psi}+\beta \mathbf{I}\right)^{-1} \boldsymbol{\psi}^{H},
$$

where $\beta$ is real valued and greater than zero.

(d) Reduced rank pseudoinverse mode filter. Let the singular value decomposition of $\boldsymbol{\psi}$ be

$$
\boldsymbol{\psi}=\mathbf{U S V}^{T}
$$

where $\mathbf{S}$ is a diagonal matrix consisting of the singular values of $\boldsymbol{\psi}$, and $\mathbf{U}$ and $\mathbf{V}$ are orthogonal matrices representing the eigenvectors of $\boldsymbol{\psi} \boldsymbol{\psi}^{T}$ and $\boldsymbol{\psi}^{T} \boldsymbol{\psi}$, respectively. The reduced rank pseudoinverse mode filter can be written as

$$
\mathbf{H}_{R R}=\boldsymbol{\psi}^{+}=\mathbf{V S}^{+} \mathbf{U}^{T},
$$

where $\mathbf{S}^{+}$is the pseudoinverse of the diagonal matrix $\mathbf{S}$, where the inverses of diagonal elements less than some threshold $(1 / 100$ of the maximum diagonal value for this paper) are set to zero. ${ }^{5}$

(e) MAP mode filter.

$$
\mathbf{H}_{\mathrm{MAP}}=\left(\mathbf{R}_{\mathbf{d}}{ }^{-1}+\boldsymbol{\psi}^{H} \mathbf{R}_{\mathbf{n}}^{-1} \boldsymbol{\psi}\right)^{-1} \boldsymbol{\psi}^{H} \mathbf{R}_{\mathbf{n}}{ }^{-1},
$$

where $\mathbf{R}_{\mathbf{d}}$ is the covariance matrix of the mode coefficients and $\mathbf{R}_{\mathbf{n}}$ is the noise covariance matrix. This assumes that the mode amplitude coefficients and noise are jointly Gaussian random variables and that the mode amplitude coefficients are independent of the noise.

\section{MINIMUM POWER DISTORTIONLESS RESPONSE MODE FILTER}

The MPDR mode filter minimizes the filter output power subject to the constraint that the desired mode is passed with a gain equal to 1 . It is based on the MPDR beamformer used in spatial array processing, with a substitution of the mode shape vector in place of the spatial steering vector. ${ }^{6-8}$ The goal is to minimize contributions from interfering signals and noise while preserving the signal propagating in the desired mode. Let the received vertical pressure field be $\mathbf{p}$ and the desired mode be $n . \mathbf{w}_{n}$ is the weight vector that filters for mode $n$, with the output of the mode filter being $\mathbf{w}_{n}^{H} \mathbf{p}$. The MPDR filter is defined by the following constrained optimization problem:

$$
\begin{aligned}
\mathbf{w}_{n} & =\underset{\mathbf{w}_{n}}{\arg \min } \mathrm{E}\left[\left|\mathbf{w}_{n}^{H} \mathbf{p}\right|^{2}\right] \\
& =\underset{\mathbf{w}_{n}}{\arg \min } \mathbf{w}_{n}^{H} \mathbf{R}_{\mathbf{p}} \mathbf{w}_{n} \text { such that } \mathbf{w}_{n}^{H} \boldsymbol{\psi}_{n}=1 .
\end{aligned}
$$

The solution is given by $y^{9,10}$

$$
\mathbf{w}_{n}=\frac{\mathbf{R}_{\mathbf{p}}^{-1} \boldsymbol{\psi}_{n}}{\boldsymbol{\psi}_{n}^{H} \mathbf{R}_{\mathbf{p}}^{-1} \boldsymbol{\psi}_{n}},
$$

where $\mathbf{R}_{\mathbf{p}}$ is the covariance matrix of the vertical pressure field at a particular frequency. In practice, $\mathbf{R}_{\mathbf{p}}$ is not known $a$ priori and must be estimated from the received data. The sample covariance matrix is one option for estimating $\mathbf{R}_{\mathbf{p}}$, but it requires a large number of snapshots to accurately estimate the matrix. The linear mode filter $\mathbf{H}$ that estimates all the modes together is formed by combining the weights for each mode: 


$$
\mathbf{H}_{\mathrm{MPDR}}=\left[\begin{array}{c}
\mathbf{w}_{1}^{H} \\
\mathbf{w}_{2}^{H} \\
\vdots \\
\mathbf{w}_{M}^{H}
\end{array}\right] .
$$

The sample covariance matrix, $\hat{\mathbf{R}}_{\text {data }}$, defined as

$$
\hat{\mathbf{R}}_{\mathrm{data}}=\frac{1}{L} \sum_{l=1, \ldots, L} \mathbf{X}_{l}(\omega) \mathbf{X}_{l}(\omega)^{H}
$$

is often used as the estimate of $\mathbf{R}_{\mathbf{p}}$, where $\mathbf{X}_{l}(\omega)$ is the array snapshot vector at frequency $\omega .{ }^{6}$ This matrix is the unconstrained maximum likelihood estimate of the covariance matrix. ${ }^{11} \hat{\mathbf{R}}_{\text {data }}$ is a good approximation of the true matrix only when a large number of snapshots are used. An inadequate number of snapshots results in poor adaptive algorithm performance. ${ }^{6,12}$

A diagonal weighting technique may be used on the sample covariance matrix to compensate for an inadequate number of snapshots and improve filter performance, similar to the diagonally weighted PI filter. ${ }^{6,13}$ When the weighting factor, $\beta$, increases by a large amount, $\hat{\mathbf{R}}_{\mathrm{DW}}$ becomes proportional to the identity matrix and the diagonally weighted MPDR filter becomes the same as the SMS filter.

$$
\hat{\mathbf{R}}_{\mathrm{DW}}=\hat{\mathbf{R}}_{\mathrm{data}}+\beta \mathbf{I} \text {. }
$$

\section{PCML METHOD}

This section describes a physically constrained, maximum likelihood method for estimating the spatial covariance matrix used in adaptive filters. This method was proposed by Kraay in 2003. ${ }^{2}$ The goal of the PCML method is to improve adaptive array processing methods under snapshot-deficient conditions. The algorithm determines the maximum likelihood estimate of the spatial covariance matrix subject to known physical constraints. The physical constraints come from our knowledge that the received signal is composed of a propagating component plus spatially white sensor noise. As a result of the physical constraints, fewer snapshots are required to obtain an accurate estimate of the spatial covariance matrix.

\section{A. PCML covariance matrix estimate}

The signal snapshots are modeled as series of independent, identically distributed (i.i.d.) zero-mean complex Gaussian random vectors (CGRVs). The joint probability density function of $L$ such snapshots is ${ }^{14}$

$$
p\left(\mathbf{X}_{l}, \ldots, \mathbf{X}_{L}\right)=\prod_{l=1, \ldots, L} \frac{1}{\pi^{N}|\mathbf{R}|} e^{-\mathbf{X}_{l}^{H} \mathbf{R}^{-1} \mathbf{x}_{l}},
$$

where $\mathbf{R}$ is the covariance matrix of those snapshots and $N$ is the number of elements in each vector $\mathbf{X}$. The ML estimate of this covariance matrix given the received snapshots is

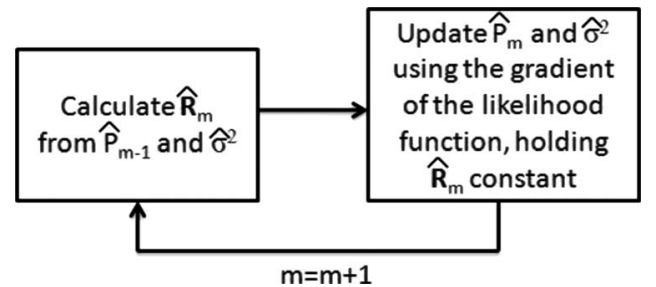

FIG. 1. Iteration used in the PCML algorithm to find the constrained maximum likelihood spatial covariance matrix estimate.

$$
\begin{aligned}
\hat{\mathbf{R}}_{\mathrm{ML}} & =\underset{\mathbf{R}}{\arg \max } p\left(\mathbf{X}_{1}, \ldots, \mathbf{X}_{L}\right) \\
& =\underset{\mathbf{R}}{\arg \max } \prod_{l=1 \ldots L} \frac{1}{\pi^{N}|\mathbf{R}|} e^{-\mathbf{X}_{l}^{H} \mathbf{R}^{-1} \mathbf{X}_{l}} \\
& =\underset{\mathbf{R}}{\arg \max } \pi^{-N L}|\mathbf{R}|^{-L} e^{-\Sigma_{l=1 \ldots L^{-}} \mathbf{X}_{l}^{H} \mathbf{R}^{-1} \mathbf{X}_{l}} \\
& =\underset{\mathbf{R}}{\arg \max }-\log |\mathbf{R}|-\frac{1}{L} \sum_{l=1, \ldots, L} \operatorname{Tr}\left(\mathbf{X}_{l}^{H} \mathbf{R}^{-1} \mathbf{X}_{l}\right) \\
& =\underset{\mathbf{R}}{\arg \max }-\log |\mathbf{R}|-\operatorname{Tr}\left(\frac{1}{L_{l=1, \ldots, L}} \sum_{\left.\mathbf{R}^{-1} \mathbf{X}_{l} \mathbf{X}_{l}^{H}\right)}\right. \\
& =\underset{\mathbf{R}}{\arg \max }-\log |\mathbf{R}|-\operatorname{Tr}\left(\mathbf{R}^{-1} \hat{\mathbf{R}}_{\mathrm{data}}\right),
\end{aligned}
$$

where $\quad L\left(\mathbf{R}, \hat{\mathbf{R}}_{\text {data }}\right)=-\log |\mathbf{R}|-\operatorname{Tr}\left(\mathbf{R}^{-1} \hat{\mathbf{R}}_{\text {data }}\right) \quad$ is the $\log$ likelihood function. There is no closed form solution for the $\mathbf{R}$ that maximizes this log-likelihood function when the physical constraint of Eq. (20) is imposed. It is, however, possible to use derivatives of the likelihood function in an iterative approach to finding the constrained ML estimate. Kraay $^{2}$ derived this method for a spatial beamformer. The covariance matrix is separated into its propagating component plus spatially white sensor noise,

$$
[\mathbf{R}]_{i j}=\sigma^{2} \delta_{i j}+\frac{1}{(2 \pi)^{3}} \int_{\Omega(\mathbf{k})} P(\omega, \mathbf{k})[\mathbf{v}(\mathbf{k})]_{i}\left[\mathbf{v}^{\mathbf{H}}(\mathbf{k})\right]_{j} d \mathbf{k},
$$

where $[\mathbf{v}(\mathbf{k})]_{n}=e^{-j \mathbf{k}^{T} \mathbf{p}_{n}}$ is the $n$th element of the array manifold vector, $\mathbf{p}_{n}$ is the location of the $n$th array element, $\mathbf{k}$ is the spatial wavenumber vector, and $P(\omega, \mathbf{k})$ is the frequency wavenumber power spectrum. $\Omega(\mathbf{k})$ is the region of support of the wavenumber field imposed by the wave equation, $\|\mathbf{k}\|=2 \pi / \lambda=\omega / c$.

\section{B. Iteration structure}

The PCML algorithm iterates between estimating $\mathbf{R}$ given current estimates of $P(\omega, \mathbf{k})$ and $\sigma^{2}$ and using the gradient of the likelihood function with respect to $P(\omega, \mathbf{k})$ and $\sigma^{2}$ to update $\hat{P}_{m}(\omega, \mathbf{k})$ and $\hat{\sigma}_{m}^{2}$, where the subscript $m$ denotes the estimate of the quantity at the $m$ th iteration. The structure is shown in Fig. 1 and is discussed in detail in Secs. III C and III E. 


\section{Initialization}

The PCML algorithm initializes its estimate of the covariance matrix with the sample covariance matrix, $\hat{\mathbf{R}}_{0}=\hat{\mathbf{R}}_{\text {data }}$. The initial frequency wavenumber spectral estimate, $P(\omega, \mathbf{k})$, is estimated using the MPDR estimator, ${ }^{8}$

$$
\begin{aligned}
\hat{P}_{0}\left(\omega, \mathbf{k}_{n}\right) & =\mathbf{w}_{\mathrm{MPDR}}^{H}\left(\mathbf{k}_{n}\right) \hat{\mathbf{R}}_{\mathrm{data}} \mathbf{w}_{\mathrm{MPDR}}\left(\mathbf{k}_{n}\right) \\
& =\frac{1}{\mathbf{v}^{H}\left(\mathbf{k}_{n}\right) \hat{\mathbf{R}}_{\mathrm{data}}^{-1} \mathbf{v}\left(\mathbf{k}_{n}\right)} .
\end{aligned}
$$

The white noise power estimate is initialized as one-tenth the average diagonal value of $\hat{\mathbf{R}}_{\text {data }}$ since it is physically restricted to lie between zero and the smallest diagonal value of $\hat{\mathbf{R}}_{\text {data. }}$.

$$
\hat{\sigma}_{0}^{2}=\frac{1}{10 N} \operatorname{Tr}\left(\hat{\mathbf{R}}_{\text {data }}\right) .
$$

\section{Covariance matrix update}

The first iterative step is to obtain a new estimate of the covariance matrix. This is done by inverse Fourier transforming the frequency wavenumber spectrum with respect to the wavenumber vector $\mathbf{k}$.

$$
\begin{aligned}
{\left[\hat{\mathbf{R}}_{m}\right]_{i j}=} & {\left[\mathcal{F}^{-1}\left(\hat{P}_{m-1}(\omega, \mathbf{k})+\hat{\sigma}_{m-1}^{2}\right)\right]_{i, j}=\hat{\sigma}_{m-1}^{2} \delta_{i j} } \\
& +\frac{1}{(2 \pi)^{3}} \int_{\Omega(\mathbf{k})} \hat{P}_{m-1}(\omega, \mathbf{k}) e^{-j \mathbf{k}^{T}\left(\mathbf{p}_{i}-\mathbf{p}_{j}\right)} d \mathbf{k},
\end{aligned}
$$

where $\hat{P}_{m-1}(\omega, \mathbf{k})$ is the estimate of the power at frequency $\omega$ coming from the direction specified by $\mathbf{k}$ at iteration $m-1$. Since $\hat{P}_{m}$ can only be calculated at discrete points, a covariance matrix taper is used to smooth the estimates of propagating energy around the discrete spatial plane wave samples. Each sample, $\hat{P}_{m-1}(\omega, \mathbf{k})$, is approximated as a weighted, shifted window in $\mathbf{k}$-space,

$$
\hat{P}_{m-1}(\omega, \mathbf{k})=\sum_{n}\left[\hat{P}_{m-1}\left(\omega, \mathbf{k}_{n}\right) W\left(\mathbf{k}-\mathbf{k}_{n}\right)\right],
$$

where $\mathbf{k}_{n}$ is the $n$th sample of $\mathbf{k}$ at which the frequency wavenumber spectrum is calculated. The integral in Eq. (23) then becomes a summation,

$$
\left[\hat{\mathbf{R}}_{m}\right]_{i j}=\hat{\sigma}_{m-1}^{2} \delta_{i j}+\left(\sum_{n} \hat{P}_{m-1}\left(\omega, \mathbf{k}_{n}\right) e^{-j \mathbf{k}_{n}^{T}\left(\mathbf{p}_{i}-\mathbf{p}_{j}\right)}\right) \mathbf{W}_{i j}
$$

where $\mathbf{W}_{i j}$ is the inverse Fourier transform of the covariance matrix taper. ${ }^{15,16}$ The taper primarily used by Kraay was a uniform window given by ${ }^{13}$

$$
\begin{aligned}
W(\mathbf{u}) & =W\left(u_{x}, u_{y}\right) \\
& =\left\{\begin{array}{lr}
1 & \text { for }\left|u_{x}\right|<\Delta u / 2 \text { and }\left|u_{y}\right|<\Delta u / 2 \\
0 & \text { otherwise, }
\end{array}\right.
\end{aligned}
$$

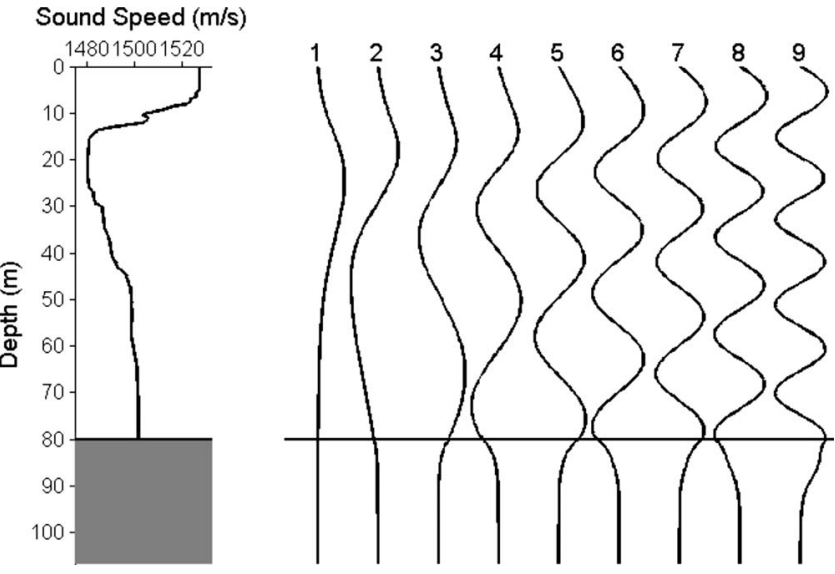

FIG. 2. Shallow water sound speed profile and mode shapes at $200 \mathrm{~Hz}$.

where $\mathbf{u}$ is the wavenumber vector, normalized by $2 \pi / \lambda$, and $\Delta u$ is the wavenumber grid spacing. The inverse Fourier transform of this window is

$$
\begin{aligned}
\mathbf{W}_{i, j}= & \int W(\mathbf{u}) e^{+j(2 \pi / \lambda) \mathbf{u}^{T}\left(\mathbf{p}_{i}-\mathbf{p}_{j}\right)} d \mathbf{u} \\
= & \Delta u \operatorname{sinc}\left(\frac{2 \pi}{\lambda} \frac{\Delta u}{2}\left(p_{i, x}-p_{j, x}\right)\right) \Delta u \operatorname{sinc} \\
& \times\left(\frac{2 \pi}{\lambda} \frac{\Delta u}{2}\left(p_{i, y}-p_{j, y}\right)\right) .
\end{aligned}
$$

\section{E. Power spectrum and noise power updates}

The power spectral density estimates of the power in each mode are updated using the gradient of the likelihood function [Eq. (19)] with respect to the power estimates.

The gradient with respect to the power at a given wavenumber is given by

$$
\begin{aligned}
\frac{\partial L\left(\hat{\mathbf{R}}_{m}, \hat{\mathbf{R}}_{\text {data }}\right)}{\partial \hat{P}_{m}\left(\omega, \mathbf{k}_{n}\right)}= & -\mathbf{v}^{H}\left(\mathbf{k}_{n}\right) \hat{\mathbf{R}}_{m}^{-1} \mathbf{v}\left(\mathbf{k}_{n}\right) \\
& +\mathbf{v}^{H}\left(\mathbf{k}_{n}\right) \hat{\mathbf{R}}_{m}^{-1} \hat{\mathbf{R}}_{\text {data }} \hat{\mathbf{R}}_{m}^{-1} \mathbf{v}\left(\mathbf{k}_{n}\right)
\end{aligned}
$$

and the gradient with respect to the white noise power is given by

$$
\frac{\partial L\left(\hat{\mathbf{R}}_{m}, \hat{\mathbf{R}}_{\text {data }}\right)}{\partial \hat{\sigma}_{m}^{2}}=\operatorname{Tr}\left(\left(\hat{\mathbf{R}}_{m}^{-1} \hat{\mathbf{R}}_{\text {data }}-\mathbf{I}\right) \hat{\mathbf{R}}_{m}^{-1}\right) .
$$

The second order gradient can be used to verify that a maximum of the likelihood function is reached and not a minimum. The multiplicative update proposed by Kraay provides a convenient mapping between the gradients of the likelihood function and a multiplicative scaling factor. The update is chosen such that when the gradient of the likelihood function is zero, the power estimates remain unchanged. The scale factor increases monotonically for a positive gradient and decreases monotonically for a negative gradient. 


$$
\hat{P}_{m}\left(\omega, \mathbf{k}_{n}\right)= \begin{cases}\hat{P}_{m-1}\left(\omega, \mathbf{k}_{n}\right)\left[\frac{A-1}{e^{\pi / 2}-1}\left(e^{\arctan \left(\alpha \partial L /\left(\partial \hat{P}_{m-1}\left(\omega, \mathbf{k}_{n}\right)\right)\right)}-1\right)+1\right], & \frac{\partial L}{\partial \hat{P}_{m-1}}>0 \\ \hat{P}_{m-1}\left(\omega, \mathbf{k}_{n}\right)\left[\frac{B-1}{e^{-\pi / 2}-1}\left(e^{\arctan \left(\beta \partial L /\left(\partial \hat{P}_{m-1}\left(\omega, \mathbf{k}_{n}\right)\right)\right)}-1\right)+1\right] & \text { otherwise }\end{cases}
$$

where $A$ and $B$ are the scale's upper and lower limits, and $\alpha$ and $\beta$ are parameters that control how quickly the algorithm steps as a function of the gradient. For the white noise power update, Kraay used an additive form since it offered better stability in her environment.

$$
\hat{\sigma}_{m}^{2}=\hat{\sigma}_{m-1}^{2}+10^{-4} \frac{\partial L}{\partial \hat{\sigma}_{m-1}^{2}} /\left|\frac{\partial^{2} L}{\left(\partial \hat{\sigma}_{m-1}^{2}\right)^{2}}\right| .
$$

The PCML algorithm iterates for a number of iterations until the covariance matrix estimate has converged to its most likely value. The PCML frequency wavenumber spectrum estimates are the values of $\hat{P}_{m}(\omega, \mathbf{k})$ at the final iteration of the algorithm. The likelihood function can be calculated at each iteration to provide an indication of whether the algorithm has converged, and a stopping condition can be formed based on this. Kraay chose to run the algorithm for 50 iterations.

\section{PCML ALGORITHM APPLIED TO MODES}

This section describes the application of Kraay's PCML algorithm to the problem of estimating complex-valued mode amplitudes. For the underwater environment model of Eq. (1), the covariance matrix of the acoustic pressure field can be decomposed into a propagating modal component plus spatially white sensor noise. In this case, there are a discrete number of propagating modes to sum instead of an infinite number of spatial plane waves to integrate over, so no covariance matrix taper is necessary. Thus Eqs. (23) and (25) become

$$
\hat{\mathbf{R}}=\hat{\sigma}^{2} \mathbf{I}+\sum_{n=1, \ldots, M} \hat{P}\left(\omega, \boldsymbol{\psi}_{n}\right) \boldsymbol{\psi}_{n} \boldsymbol{\psi}_{n}^{H} .
$$

The second change is that instead of steering the beamformer to a spatial direction, it is steered to a particular mode. The array steering vector, $\mathbf{v}(\mathbf{k})$, becomes

$$
\mathbf{v}\left(\mathbf{k}_{n}\right)=\psi_{n} .
$$

With these modifications, the PCML algorithm developed by Kraay can be applied to determine the maximum likelihood estimate of the covariance matrix given the physical constraint. With each iteration, the PCML algorithm generates an estimate of the power in each mode and the power of the spatially white noise. These estimates are used to generate an estimate of the covariance matrix using Eq. (32). Once the PCML algorithm has converged, the PCML-MPDR filter uses the estimate of the covariance matrix at the final iteration of the PCML algorithm in the MPDR filter, Eqs. (14) and (15). The PCML-MAP filter implements a MAP mode filter using the estimates of the signal and noise statistics from the final iteration of the PCML algorithm. That is, the filter assumes that the mode amplitude coefficients are uncorrelated, and the noise is spatially white and calculates

$$
\mathbf{R}_{\mathbf{d}}=\left[\begin{array}{cccc}
\hat{P}\left(\omega, \boldsymbol{\psi}_{1}\right)^{2} & 0 & \cdots & 0 \\
0 & \hat{P}\left(\omega, \boldsymbol{\psi}_{2}\right)^{2} & \ddots & \vdots \\
\vdots & \vdots & \ddots & 0 \\
0 & \cdots & 0 & \hat{P}\left(\omega, \boldsymbol{\psi}_{M}\right)^{2}
\end{array}\right]
$$

and

$$
\mathbf{R}_{\mathbf{n}}=\hat{\sigma}^{2} \mathbf{I}
$$

in Eq. (12), using $\hat{P}$ and $\hat{\sigma}^{2}$ from the final PCML iteration.

\section{PERFORMANCE AND ANALYSIS}

\section{A. Simulation setup}

This section describes the setup of the shallow water simulation. The mode coefficient vector, $\mathbf{d}$, is modeled as a zero-mean complex Gaussian random vector. The noise is modeled as spatially white. The real and imaginary parts of $\mathbf{d}$ and $\mathbf{n}$ are i.i.d. and Gaussian, and therefore their covariance matrices are real valued. ${ }^{14}$ The simulation is similar to the one described by Buck et al. ${ }^{1}$ for a shallow water environment. The simulated environment had typical shallow water sound speed profile and bottom properties and was $80 \mathrm{~m}$ deep. Figure 2 shows the sound speed profile and the corresponding mode shapes at a frequency of $200 \mathrm{~Hz}$.

A vertical array of 20 equally spaced hydrophones was used. The location of the bottom hydrophone was fixed at 79 $\mathrm{m}$ depth and the depth of the top hydrophone was varied from the water surface to a depth of $40 \mathrm{~m}$ (half the water column). This gradually reduced the fraction of the water column that was spanned by the array. For each aperture, 500 trials were run using independent realizations of the mode coefficients and noise vector. Linear mode filters were applied to the simulated pressure field to obtain an estimate of the mode amplitudes. The error criteria are the sample mean squared errors, $\|\hat{\mathbf{d}}-\mathbf{d}\|^{2}$, between the estimate of the complex mode amplitudes and their actual values over the 500 trials. The total mode energy is included in the plots for reference and represents the error that would result from estimating each mode to have zero amplitude. The mode coefficients, $\mathbf{d}$, were assumed to be an i.i.d., zero-mean, CGRV. Except where stated otherwise, 25 snapshots were used to initialize the sample covariance matrix for the PCML algorithms and for the MPDR filter. 


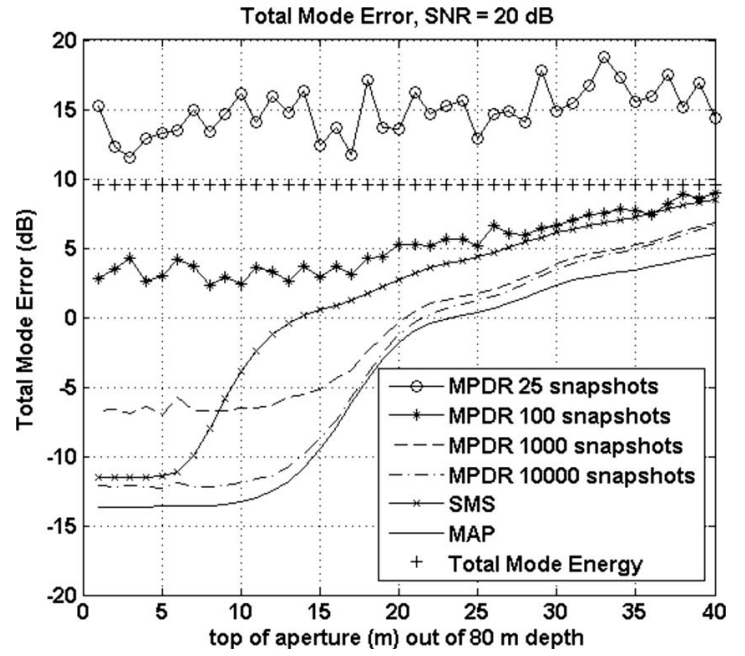

FIG. 3. Comparison of the performance of the MPDR mode filters as the number of snapshots used in the sample covariance matrix is varied. The SMS and MAP filter performances and the total mode energy are included for reference.

\section{B. Simulation results}

Figure 3 shows the performance of the MPDR filter using the sample covariance matrix as the number of snapshots is varied. As was discussed in Sec. II, thousands of snapshots are required for the covariance matrix to converge to its final value. When sufficient snapshots are used, the MPDR filter's performance matches that of the MAP filter for a full spanning array, but is a few $\mathrm{dB}$ worse than the MAP filter when the array span is reduced.

Figure 4 shows the effect of changing the loading parameter on the diagonally weighted PI filter. With only small amounts of weighting, the filter still suffers from sensitivity to white noise as the span of the array is reduced. With large amounts of weighting, the filter performs poorly, as the amplitude of the mode estimates approaches zero. Only when the correct weighting is applied is the filter able to perform the same as the MAP filter.

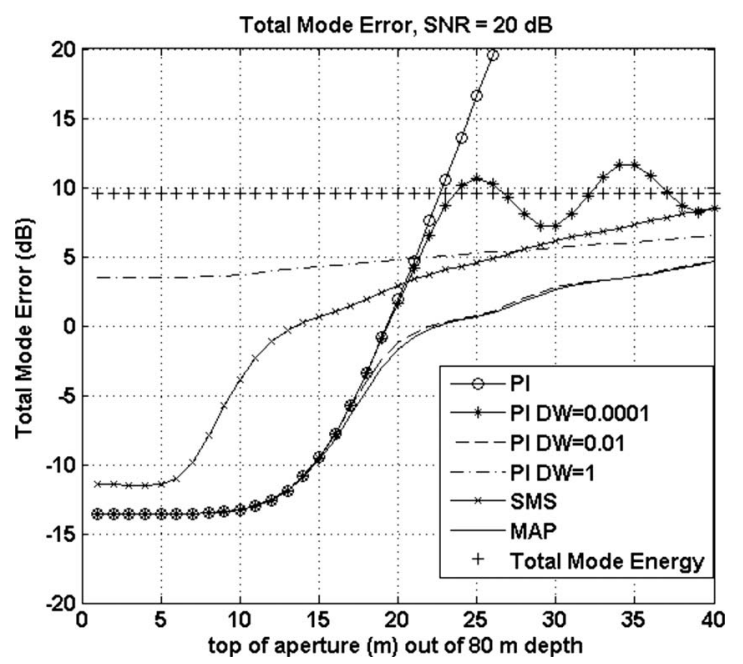

FIG. 4. Comparison of the performance of the diagonally weighted PI filter as the weighting factor is varied. The SMS and MAP filter performances and the total mode energy are included for reference.

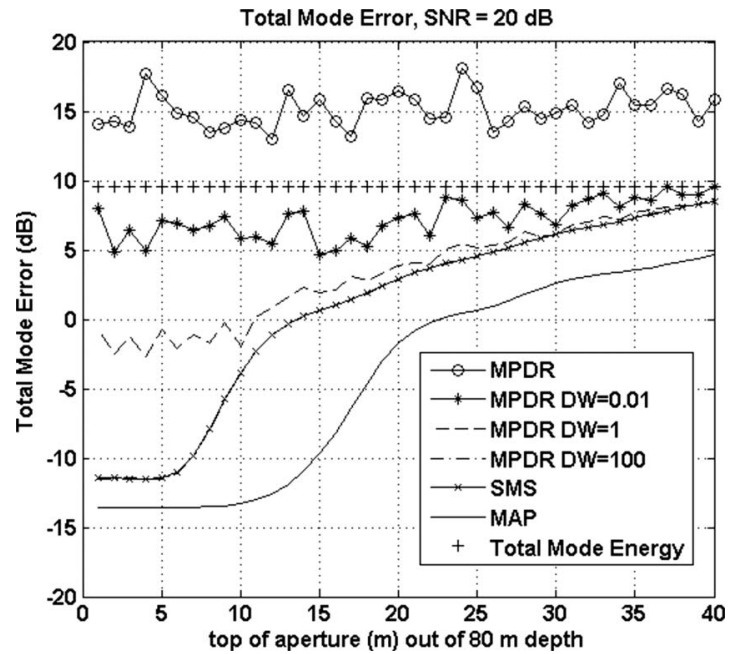

FIG. 5. Comparison of the performance of the diagonally weighted MPDR filter as the weighting factor is varied, using 25 snapshots to generate the sample covariance matrix in the MPDR filters. The SMS and MAP filter performances and the total mode energy are included for reference. With a diagonal weighting of 100 , the MPDR curve falls on top of the SMS curve.

Figure 5 shows the effect of changing the loading parameter on the diagonally weighted MPDR filter. The filter converges on the SMS filter as the weighting is increased; however, it is unable to perform better than SMS.

Figure 6 shows the simulation results for the PCMLMAP, PCML-MPDR, and reduced rank PI filters. With only 25 snapshots, the PCML-MPDR filter is able to do as well as the unconstrained MPDR filter using 10000 snapshots. Furthermore, the PCML-MAP filter performs as well as the MAP filter that has full knowledge of the signal and noise statistics. The sawtooth pattern in the reduced rank PI filter is a result of the changing number of singular values used in the pseudoinversion as the condition number of $\psi$ changes. As the condition number worsens, singular values are dropped one at a time resulting in the observed pattern.

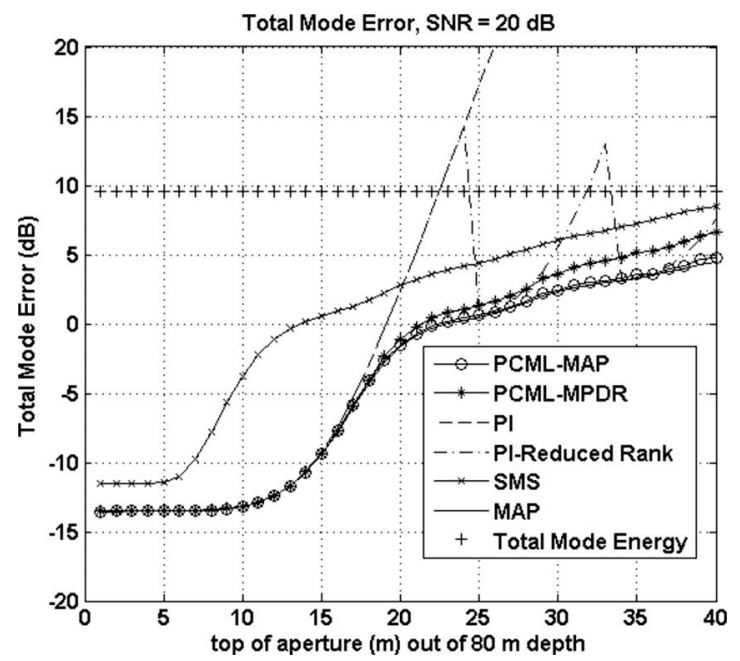

FIG. 6. Comparison of the performance of the PCML-MAP, PCML-MPDR, PI, and reduced rank PI filters. The SMS and MAP filter performances and the total mode energy are included for reference. The PCML algorithms were initialized with 25 data snapshots. 


\section{CONCLUSIONS}

The MPDR filter using the sample covariance matrix requires a large number of snapshots in order to estimate the received signal covariance matrix, $\mathbf{R}_{\mathbf{p}}$, with accuracy sufficient for the algorithm to yield satisfactory results. While diagonal loading of the sample covariance matrix can compensate for an insufficient number of snapshots, the performance of the diagonally loaded PI and MPDR algorithms is sensitive to the choice of the loading parameter. This sensitivity is undesirable in real world applications.

The PCML algorithm uses physical constraints to estimate $\mathbf{R}_{\mathbf{p}}$ and the second order statistics of the mode amplitudes and noise. When the resulting $\mathbf{R}_{\mathbf{p}}$ is then used in a MPDR mode filter, it is found that the snapshot requirement can be reduced by over two orders of magnitude without sacrificing the resulting algorithm performance. Furthermore, using the same number of snapshots as used for the PCMLMPDR algorithm, the estimated second order signal and noise statistics can be used in the MAP mode filter and achieve the same performance as the MAP mode filter that has a priori knowledge of these statistics.

\section{ACKNOWLEDGMENTS}

This paper is based on a thesis submitted in partial fulfillment of the requirements of the degree of Master of Science in the Department of Electrical Engineering and Computer Science at the Massachusetts Institute of Technology and Woods Hole Oceanographic Institution in September, 2009. This work was supported by the Office of Naval Research through ONR Grant Nos. N00014-05-10085 and N00014-06-10788 and through the WHOI Academic Programs Office.
${ }^{1}$ J. R. Buck, J. C. Preisig, and K. E. Wage, "A unified framework for mode filtering and the maximum a posteriori mode filter," J. Acoust. Soc. Am. 103, 1813-1824 (1998).

${ }^{2}$ A. L. Kraay, "Physically constrained maximum-likelihood method for snapshot-deficient adaptive array processing," Master Thesis, Massachusetts Institute of Technology, Cambridge, MA (2003).

${ }^{3}$ F. B. Jensen, W. A. Kuperman, M. B. Porter, and H. Schmidt, Computational Ocean Acoustics (Springer-Verlag, New York, 2000).

${ }^{4}$ W. A. Kuperman and F. Ingenito, "Spatial correlation of surface generated noise in a stratified ocean," J. Acoust. Soc. Am. 67, 1988-1996 (1980).

${ }^{5}$ G. Strang, Linear Algebra and Its Applications, 4th ed. (Thompson Brooks/Cole, Belmont, CA, 2006).

${ }^{6}$ H. L. VanTrees, Optimum Array (Wiley-Interscience, New York, 2002), Part IV.

${ }^{7}$ B. D. Vanveen and K. Buckley, "Beamforming: A versatile approach to spatial filtering," IEEE ASSP Magazine (1988).

${ }^{8}$ J. Capon, "High-resolution frequency-wavenumber spectrum analysis," Proc. IEEE 57, 1408-1418 (1969).

${ }^{9} \mathrm{O}$. L. Frost, "An algorithm for linearly constrained adaptive array processing,” Proc. IEEE 60, 926-935 (1972).

${ }^{10}$ D. H. Brandwood, "A complex gradient operator and its application in adaptive array theory," IEE Proc. F, Commun. Radar Signal Process. 130, 11-16 (1983).

${ }^{11}$ J. R. Guerci, Space-Time Adaptive Processing for Radar (Artech House, Inc., Norwood, MA, 2003).

${ }^{12}$ A. B. Baggeroer and H. Cox, "Passive sonar limits upon nulling multiple moving ships with large aperture arrays," Conference Record of the 33rd Asilomar Conference on Signals, Systems, Computers (1999), Vol. 1, pp. 103-108.

${ }^{13}$ A. L. Kraay and A. B. Baggeroer, "A physically constrained maximumlikelihood method for snapshot-deficient adaptive array processing," IEEE Trans. Signal Process. 55, 4048-4063 (2007).

${ }^{14} \mathrm{D}$. Tse and P. Viswanath, Fundamentals of Wireless Communication (Cambridge University Press, New York, 2005).

${ }^{15}$ J. R. Guerci, "Theory and application of covariance matrix tapers for robust adaptive beamforming," IEEE Trans. Signal Process. 47, 977-985 (1999).

${ }^{16} \mathrm{~F}$. J. Harris, "On the use of windows for harmonic analysis with the discrete Fourier transform,” Proc. IEEE 66, 51-83 (1978). 